Check for updates

Cite this: RSC Adv., 2018, 8, 13112

\title{
Weavable asymmetric carbon nanotube yarn supercapacitor for electronic textiles $\uparrow$
}

\author{
Changsoon Choi,,$_{+}^{\mathrm{ab}}$ Jong Woo Park, $\stackrel{t}{\mathrm{t}}^{\mathrm{a}}$ Keon Jung Kim, ${ }^{a}$ Duck Weon Lee, ${ }^{a}$ \\ Mônica Jung de Andrade, ${ }^{c}$ Shi Hyeong Kim, ${ }^{c}$ Sanjeev Gambhir, ${ }^{d}$ Geoffrey M. Spinks, ${ }^{d}$ \\ Ray H. Baughman ${ }^{c}$ and Seon Jeong Kim (D) *a
}

\begin{abstract}
Asymmetric supercapacitors are receiving much research interests due to their wide operating potential window and high energy density. In this study, we report the fabrication of asymmetrically configured yarn based supercapacitor by using liquid-state biscrolling technology. High loading amounts of reduced graphene oxide anode guest (90.1 wt\%) and $\mathrm{MnO}_{2}$ cathode guest (70 wt\%) materials were successfully embedded into carbon nanotube yarn host electrodes. The resulting asymmetric yarn supercapacitor coated by gel based organic electrolyte (PVDF-HFP-TEA.BF 4 ) exhibited wider potential window (up to $3.5 \mathrm{~V})$ and resulting high energy density $\left(43 \mu \mathrm{W} \mathrm{h} \mathrm{cm}^{-2}\right)$. Moreover, the yarn electrodes were mechanically strong enough to be woven into commercial textiles. The textile supercapacitor exhibited stable electrochemical energy storage performances during dynamically applied deformations.
\end{abstract}

Received 13th February 2018

Accepted 1st April 2018

DOI: 10.1039/c8ra01384e

rsc.li/rsc-advances

stored electrical energy is given by $E=1 / 2 C V^{2}$, both strategies are important.

Environmentally friendly, cost-effective, highly performing metal oxides $\left(\mathrm{MnO}_{2}\right)^{\mathbf{8}, 9}$ or various conducting polymers (e.g., poly(3,4-ethylenedioxythiophene) (PEDOT), polyaniline (PANI), polypyrrole (PPy) $)^{2,10-14}$ have been extensively studied as pseudocapacitive additives to dramatically improve the charge storage capability of 1D supercapacitors. Asymmetrically configured 1D supercapacitors used active materials like graphene, carbon nanotubes (CNTs), and PPy for anode yarns and materials like $\mathrm{MnO}_{2}, \mathrm{MoS}_{2}, \mathrm{Ni}(\mathrm{OH})_{2}, \mathrm{Co}_{3} \mathrm{O}_{4}$ for cathode yarns, resulting in voltage windows between $1.5 \mathrm{~V}$ and $1.8 \mathrm{~V} .^{15-22}$

In this study, we realized fiber supercapacitors having both high specific capacitances and increased potential windows. The first utilized strategy was to trap pseudocapacitive guest materials within vascular, high electrical conductivity networks of twist-spun CNT yarns, which maximized the weight percent of the guest without significantly hindering accessibility of the electrolyte to the guest. The second strategy was to use an asymmetric electrode configuration that comprised a $\mathrm{MnO}_{2}$ containing cathode yarn and a reduced graphene oxide (rGO) containing anode yarn.

Specifically, both anode and cathode yarns were made using a novel liquid-state biscrolling technology, which embedded $90 \mathrm{wt} \% \mathrm{rGO}$ flakes as the active material in the anode yarn and $70.5 \mathrm{wt} \% \mathrm{MnO}_{2}$ nanoparticles as the active material in the cathode yarn. Despite the low weight percent of the CNT host, these guests were firmly trapped in the scrolled CNT galleries of this host. This device design resulted in an aqueous-gelelectrolyte-coated, solid-state, asymmetric supercapacitor that had a $2.1(\mathrm{~V})$ working potential and a high areal energy density 
of $30.1 \mu \mathrm{W} \mathrm{h} \mathrm{cm}{ }^{-2}$. Moreover, use of an organic gel electrolyte coating on the electrode yarns, extended the working voltage range up to $3.5 \mathrm{~V}$, and increased the areal energy density up to $43 \mu \mathrm{W} \mathrm{h} \mathrm{cm}{ }^{-2}$, which is much higher than previously reported for asymmetric yarn supercapacitors. Despite the high loading of brittle pseudocapacitive materials, the electrolyte-coated yarns could be inserted into a knitted structure textile. A textile patch, comprising eight woven supercapacitor yarns that were connected in-series or in-parallel, was used to light a blue LED for 300 seconds.

Fig. 1A schematically illustrates a solid-state, asymmetric yarn supercapacitor comprising anode and cathode CNT yarns containing embedded rGO flake and $\mathrm{MnO}_{2}$, respectively. These guest-embedded electrodes were prepared by using a powerful technology called biscrolling. ${ }^{23}$ CNT sheet stacks for the yarn host were drawn from a forest of vertically aligned CNT forest that was fabricated by chemical vapor deposition. Electrochemically active guest materials (rGO flake and $\mathrm{MnO}_{2}$ for anode and cathode, respectively) were dispersed in ethanol solution and drop casted onto a stack of CNT sheet ribbons. Twist insertion into guest-covered sheet ribbon stacks provided the biscrolled yarn electrodes which contain embedded guest materials. The loading level of active guest materials was roughly controlled by adjusting particle concentration in the dispersion.

Scanning electron microscope (SEM) images of $90 \mathrm{wt} \% \mathrm{rGO}$ embedded yarn surface and its cross-section are shown in Fig. 1B and C, respectively. The rGO flakes were obtained from chemically reduced graphene oxide sheet, as previously reported. ${ }^{24}$ Although most of the yarn guest is biscrolled into the yarn volume, the structure of guest on the yarn surface provides some indication of the guest structure within the yarn. The indicated flower-like crumbled structure surface of the rGO will enhance the diffusion rate within the yarn and avoid platelet stacking, which would reduce the surface area that is available during fast charge and discharge. SEM images of $70 \mathrm{wt} \% \mathrm{MnO}_{2}$ embedded yarn surface and cross section are also shown in Fig. 1D and E, respectively.

The electrochemical performance of a symmetric-electrode, electrochemical capacitor is shown in Fig. 2, wherein each yarn is a biscrolled yarn electrode containing $90.1 \mathrm{wt} \% \mathrm{rGO}$ flakes, which is over coated by and infiltrated with an aqueous polyvinyl alcohol (PVA)-LiCl gel electrolyte. Rectangular cyclic voltammetry (CV) curves (from 10 to $100 \mathrm{mV} \mathrm{s}^{-1}$ voltage scan rate) and triangular galvanostatic charge/discharge curves (from 0.5 to $5 \mathrm{~mA} \mathrm{~cm} \mathrm{~cm}^{-2}$ current density) were obtained, as shown in Fig. 2A and B, respectively. The CV curves did not show any peaks that can be associated with a faradic redox reaction. Linear and areal capacitances, normalized to the total length and external surface area of a single electrode, are shown as a function of versus scan rate in Fig. 2C. The length and surface area of single GO biscrolled yarn electrode were $1 \mathrm{~cm}$ and $0.1 \mathrm{~cm}^{2}$, respectively. For application in textiles, the areanormalized capacitance is especially important, since it helps define how much energy can be stored in a specific textile area.

Since the areal capacitance of the biscrolled rGO yarn supercapacitor is roughly proportional to the rGO guest loading level (Fig. S2 $\dagger$ ), the highest charge storage capability was obtained from the supercapacitor yarn having the highest content of rGO (90.1 wt\%). This symmetric-electrode supercapacitor provided linear and areal capacitances of $17 \mathrm{mF} \mathrm{cm}^{-1}$ and 172 $\mathrm{mF} \mathrm{cm}^{-2}$, respectively, which were calculated from the CV curve measured at $10 \mathrm{mV} \mathrm{s}^{-1}$ scan rate. These presently realized specific capacitances normalized with respect to a single electrode are significantly higher than previously reported for allcarbon-based supercapacitors comprising coaxial CNT fibers
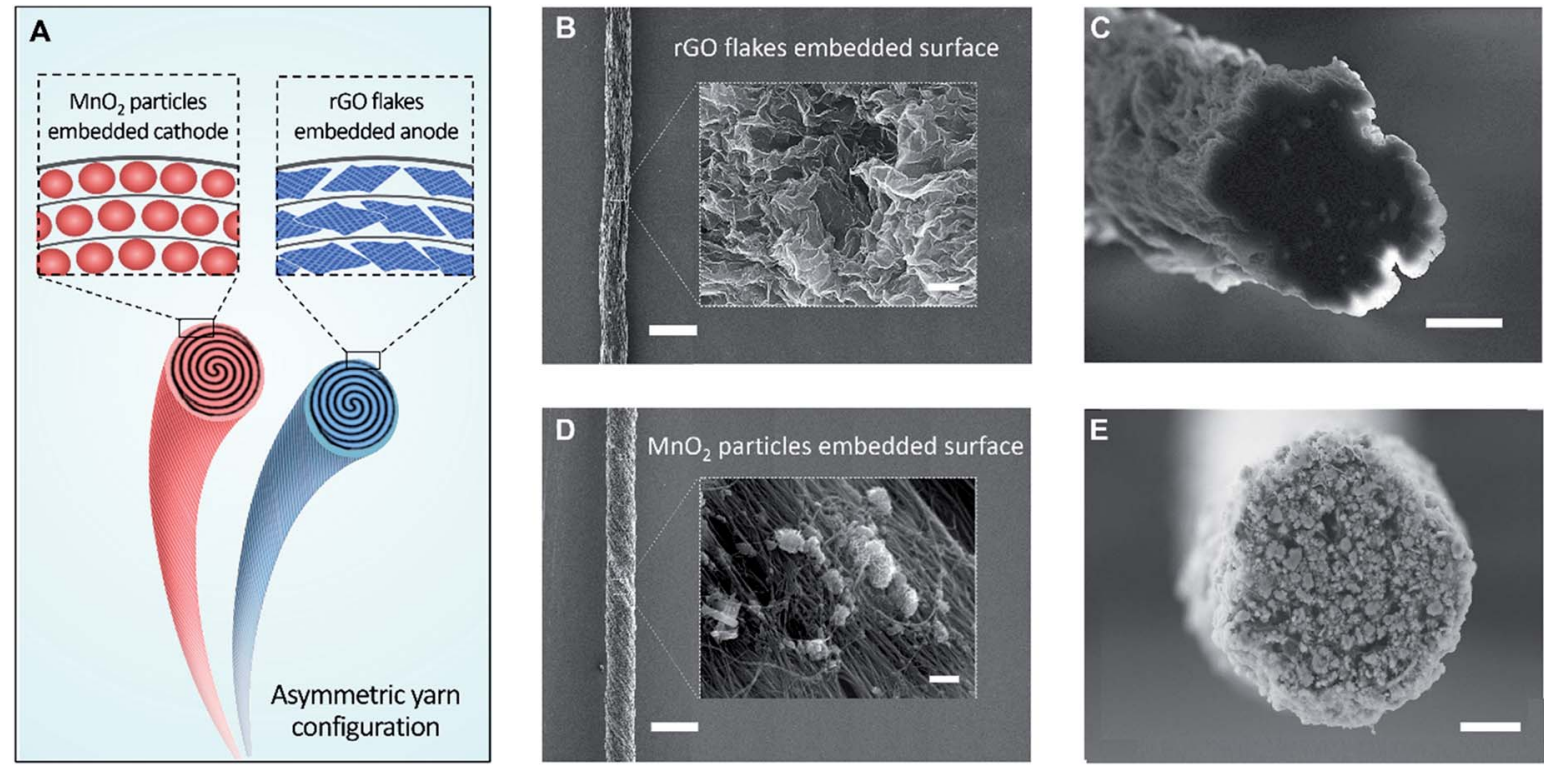

Fig. 1 (A) Illustration of a solid-state, asymmetrically configured yarn supercapacitor. Both embedded yarn electrodes were fabricated by liquidstate biscrolling process. SEM images of the surface of a $90 \mathrm{wt} \% \mathrm{rGO}$ embedded yarn anode at (B) low (scale bar $=300 \mu \mathrm{m}$ ), high magnification (inset, scale bar $=600 \mathrm{~nm}$ ), and $(\mathrm{C})$ its cross-section (scale bar $=10 \mu \mathrm{m}$ ). SEM images of the surface of a $70 \mathrm{wt}^{2} \mathrm{MnO}_{2}$ embedded yarn cathode at (D) low (scale bar $=300 \mu \mathrm{m}$ ), high magnification (inset, scale bar $=600 \mathrm{~nm}$ ), and (E) its cross-section (scale bar $=15 \mu \mathrm{m})$. 
A
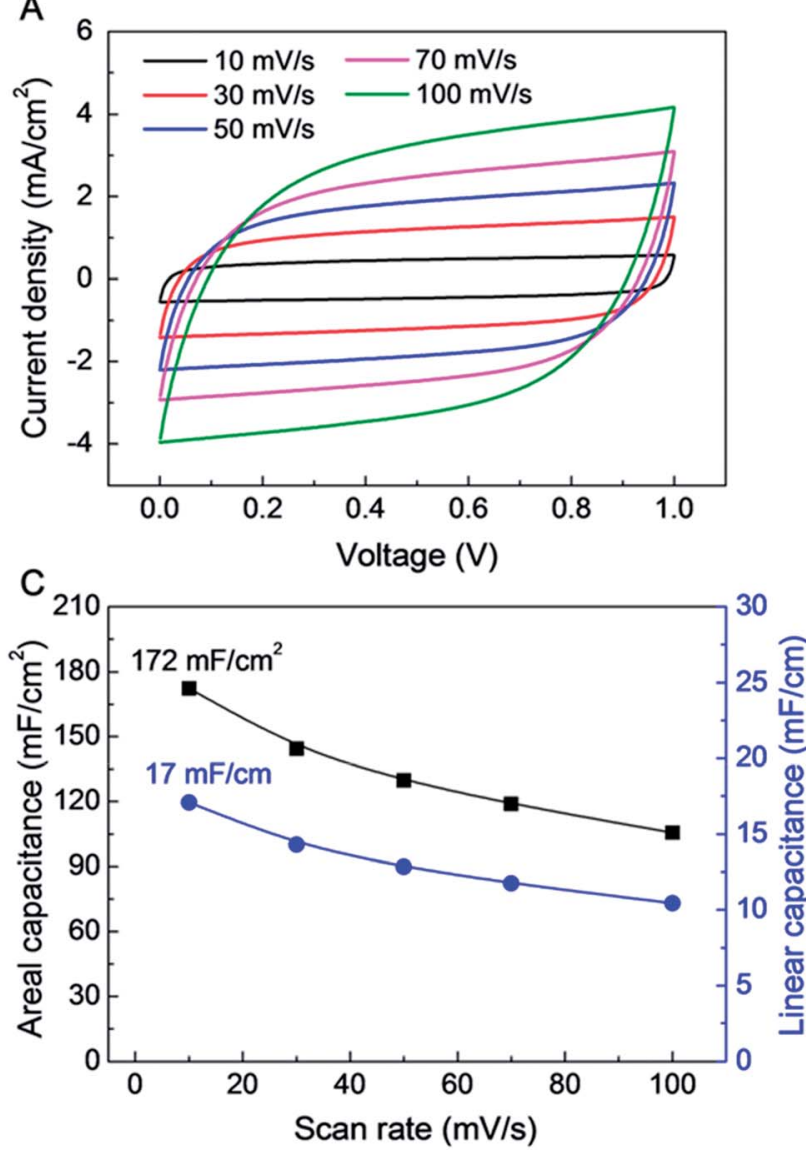

B

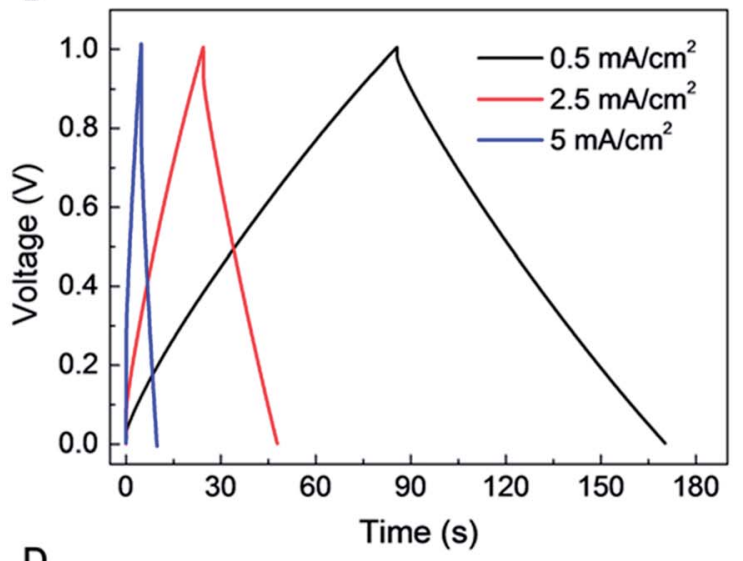

D

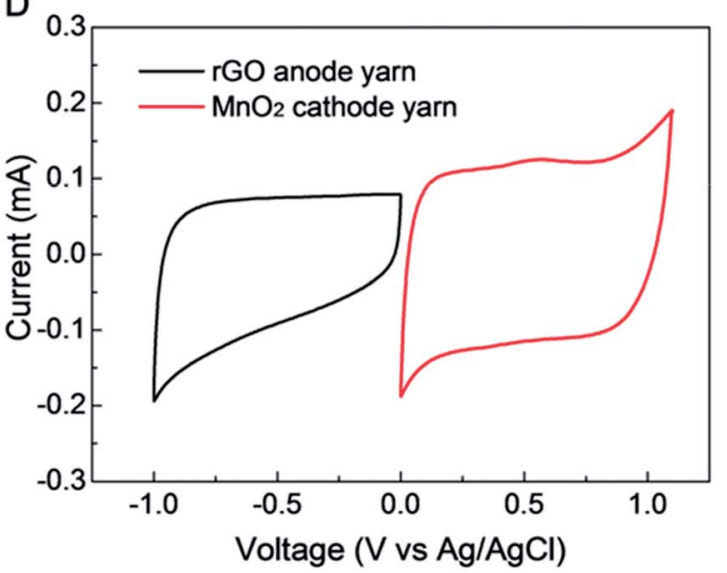

Fig. 2 Electrochemical performance of symmetric supercapacitor comprising 90 wt\% rGO embedded yarn electrodes and an aqueous PVA$\mathrm{LiCl}$ gel electrolyte. (A) CV curves measured at various scan rates (from 10 to $100 \mathrm{mV} \mathrm{s}^{-1}$ ) and (B) galvanostatic charge/discharge curves (for currents between 0.5 and $5 \mathrm{~mA} \mathrm{~cm} \mathrm{~cm}^{-2}$ ). (C) Calculated areal and linear capacitances (based on CV curves) versus scan rate. (D) Combined CV curves for anode and cathode yarns, which cover the voltage range from -1 to $1 \mathrm{~V}$. These $C \mathrm{~V}$ curves were measured in a three electrode system, using $\mathrm{Ag} / \mathrm{AgCl}$ as reference electrode and Pt mesh as counter electrode.

$\left(0.029 \mathrm{mF} \mathrm{cm}{ }^{-1}, 8.66 \mathrm{mF} \mathrm{cm}^{-2}\right),{ }^{25} \mathrm{rGO} / \mathrm{CNT}$ composite wires (0.027-0.35 mF cm $\left.{ }^{-1}, 4.97 \mathrm{mF} \mathrm{cm}^{-2}\right),{ }^{26,27}$ ordered mesoporous carbon (OMC) biscrolled fibers $\left(1.91 \mathrm{mF} \mathrm{cm}^{-1}, 39.7 \mathrm{mF} \mathrm{cm}^{-2}\right),{ }^{28}$ and even comparable coaxial wet-spun rGO/CNT composite yarns $\left(5.3 \mathrm{mF} \mathrm{cm}{ }^{-1}, 177 \mathrm{mF} \mathrm{cm}^{-2}\right) .{ }^{29}$ Such a high energy storage capability was achieved as a consequence of the high loading of rGO guest flakes that was enabled by biscrolling, as well as the structure of the crumbled structure of the rGO, which enabling them to maintain high electrochemically accessible surface area at high guest loading.

Further improvement in the performance of a yarn supercapacitor can be obtained by combining the above biscrolled rGO/CNT electrode with a biscrolled $\mathrm{MnO}_{2} / \mathrm{CNT}$ cathode ${ }^{1}$ to make an asymmetric yarn supercapacitor. To explore this opportunity, CV curves for $90 \mathrm{wt} \%$ rGO embedded anode and $70 \mathrm{wt} \% \mathrm{MnO}_{2}$ embedded cathode were measured in $0.1 \mathrm{M}$ $\mathrm{Na}_{2} \mathrm{SO}_{4}$ solution (Fig. 2D), using a three electrode system, with $\mathrm{Pt}$ mesh, and $\mathrm{Ag} / \mathrm{AgCl}$ as counter, and reference electrodes, respectively. The $\mathrm{MnO}_{2}$ loading level of $70 \mathrm{wt} \%$ was chosen to balance the charge between anode and cathode yarns when the loading level of the rGO electrode was $90 \mathrm{wt} \%$.
The electrochemical performance of an asymmetric supercapacitor comprising a $90.1 \mathrm{wt} \% \mathrm{rGO}$ embedded yarn anode, a $70 \mathrm{wt} \% \mathrm{MnO}_{2}$ embedded yarn cathode, and a PVA-LiCl based aqueous gel electrolyte coating is shown in Fig. 3. Fig. 3A shows that supercapacitor-like CV curves were obtained for voltage scans up to $2.1 \mathrm{~V}$, and there is no evidence of a faradic redox peak. Galvanostatic charge/discharge curves also showed a stable triangular shape up to $2.1 \mathrm{~V}$, with a small IR drop of $47 \mathrm{mV}$ at $1.2 \mathrm{~mA} \mathrm{~cm}^{-2}$ current density (Fig. 3B). The CV curves (0 to $2.1 \mathrm{~V}$ ) for the asymmetric supercapacitor retained a stable rectangular shape when scan rate increased from 10 to $500 \mathrm{mV} \mathrm{s}^{-1}$, retaining $61.8 \%$ initial capacitance (Fig. 3C, S4C $\dagger$ ). The volumetric and areal capacitances for this asymmetric supercapacitor (measured for up to $2.1 \mathrm{~V}$ applied potential) were $57.2 \mathrm{~F} \mathrm{~cm}^{-3}$ and $322.4 \mathrm{mF} \mathrm{cm}^{-2}$, respectively, for a voltage scan rate of $10 \mathrm{mV} \mathrm{s}^{-1}$ (Fig. S4 $\dagger$ ). Also, this asymmetric supercapacitor exhibited excellent capacitance retention during 1000 charge/discharge cycles at $200 \mathrm{mV} \mathrm{s}^{-1}$ scan rate (Fig. S5 $\dagger$ ).

For further increase of the working voltage window, we introduced a poly(vinylidenefluoride-hexafluoropropylene) (PVDF-HFP) gel-based tetraethylammonium tetrafluoroborate 

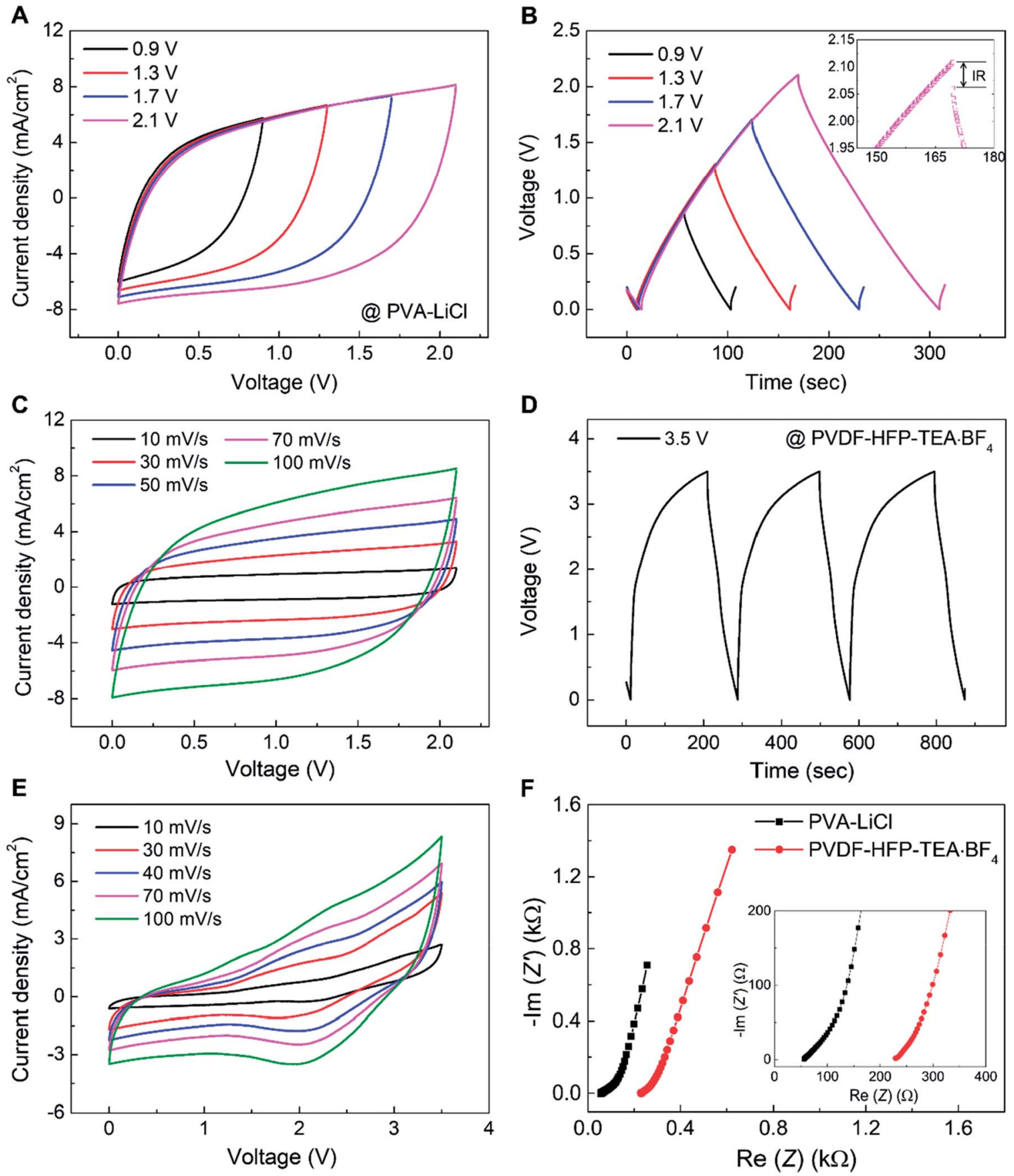

Fig. 3 Electrochemical performance of an asymmetric supercapacitor comprising a $90 \mathrm{wt} \% \mathrm{rGO}$ embedded yarn anode, a $\mathrm{MnO}_{2}$ nanoparticle embedded yarn cathode and an aqueous PVA-LiCl gel electrolyte or a polycarbonate-containing PVDF-HFP-TEA-BF 4 Organic gel electrolyte. (A) $\mathrm{CV}$ curves at $100 \mathrm{mV} \mathrm{s}^{-1}$ scan rate for differing maximum applied voltages (from $0.9 \mathrm{~V}$ to $2.1 \mathrm{~V}$ ) when using a PVA-LiCl gel electrolyte. (B) Galvanostatic-charge/discharge curves (at $1.2 \mathrm{~mA} \mathrm{~cm}^{-2}$ current density) measured over voltage window of from 0.9 to $2.1 \mathrm{~V}$. (C) CV curves measured at various scan rates $\left(10-100 \mathrm{mV} \mathrm{s}^{-1}\right.$ ) when using a PVA-LiCl gel electrolyte. (D) Galvanostatic-charge/discharge curves (at 1.6 $\mathrm{mA} \mathrm{cm}{ }^{-2}$ ) when using a PVDF-HFP-TEA $\cdot \mathrm{BF}_{4}$ based organic gel electrolyte. (E) $\mathrm{CV}$ curves up to $3.5 \mathrm{~V}$ for various voltage scan rates when using PVDF-HFP-TEA $\cdot \mathrm{BF}_{4}$ based organic gel electrolyte. (F) Comparison of results from electrochemical impedance spectroscopy when using an aqueous PVA-LiCl electrolyte and a PVDF-HFP-TEA $\cdot \mathrm{BF}_{4}$ based organic electrolyte.

$\left(\mathrm{TEA} \cdot \mathrm{BF}_{4}\right)$ organic electrolyte in propylene carbonate (PC) as a higher redox stability electrolyte for the rGO embedded yarn anode and $\mathrm{MnO}_{2}$ embedded yarn cathode asymmetric supercapacitor described above. ${ }^{\mathbf{3 0}, 31}$ The galvanostatic charge/ discharge curves showed a stable triangular shape up to $3.5 \mathrm{~V}$ for a current density of $1.6 \mathrm{~mA} \mathrm{~cm}^{-2}$ (Fig. 3D). The organic-gelelectrolyte-coated asymmetric supercapacitor was investigated over a $3.5 \mathrm{~V}$ range, for voltage scan rates from 10 to $100 \mathrm{mV} \mathrm{s}^{-1}$ 
A

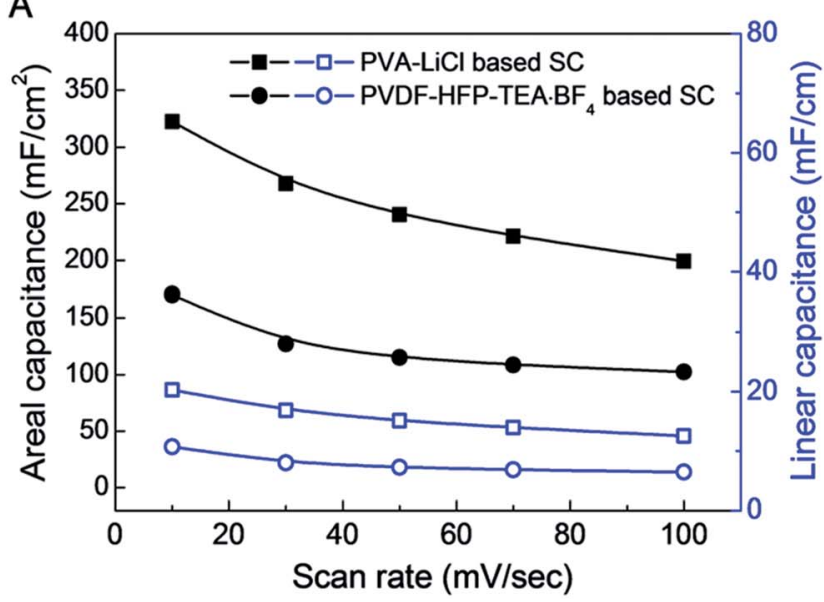

B

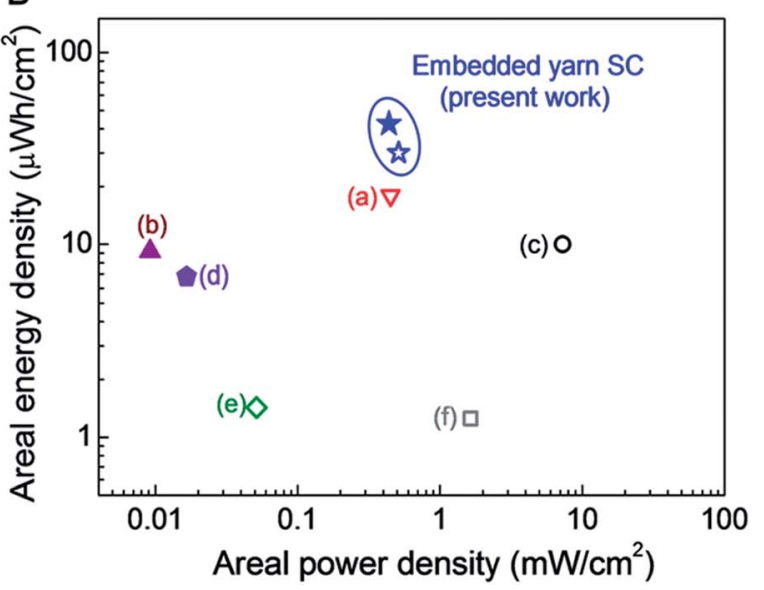

Fig. 4 (A) Areal and linear capacitances for an asymmetric supercapacitor comprising a 90 wt\% rGO embedded yarn anode and a $\mathrm{MnO}_{2}$ nanoparticle embedded yarn cathode when using either an aqueous PVA-LiCl gel electrolyte or a PVDF-HFP-TEA.BF 4 based organic gel electrolyte. (B) A Ragone plot of areal energy density versus areal power density for asymmetric $\mathrm{rGO} / \mathrm{MnO}_{2}$ embedded yarn supercapacitors, based on the total surface area of the complete supercapacitor, including embedded yarn and PVDF-HFP-TEA.BF 4 Or PVA-LiCl based gel electrolytes. The maximum measured areal energy densities of presently investigated PVDF-HFP-TEA. BF 4 organic and PVA-LiCl based aqueous gel electrolytes coated asymmetric embedded yarn supercapacitors are 30.1 and $43 \mu \mathrm{W} \mathrm{h} \mathrm{cm}{ }^{-2}$, respectively. Previously published data for assymetric yarn or fiber supercapacitors are included for comparison: (a) $\mathrm{CNT} / \mathrm{MnO}_{2}$ based stretchable asymmetric fibers (18.9 $\left.\mu \mathrm{W} \mathrm{h} \mathrm{cm}^{-2}\right),{ }^{18}$ (b) $\mathrm{rGO} / \mathrm{MnO}_{2} / \mathrm{PPy}$ yarns $\left(9.2 \mu \mathrm{W} \mathrm{h} \mathrm{cm}{ }^{-2}\right),{ }^{28}$ (c) $\mathrm{NiOH} / \mathrm{MnO}_{2}$ asymmetric yarns $\left(10 \mu \mathrm{W} \mathrm{h} \mathrm{cm}{ }^{-2}\right)$, ${ }^{12}$ (d) hollow rGO fibers $\left(6.8 \mu \mathrm{W} \mathrm{h} \mathrm{cm}^{-2}\right),{ }^{29}$ (e) $\mathrm{MnO}_{2}$ nanosheet decorated asymmetric carbon fibers $\left(1.428 \mu \mathrm{W} \mathrm{h} \mathrm{cm}{ }^{-2}\right),{ }_{17}^{17}$ and (f) $\mathrm{MnO}_{2}$ coated stretchable, asymmetric CNT wires (1.25 $\mu \mathrm{W}$ h cm $\left.{ }^{-2}\right) .{ }^{15}$

(Fig. 3E). The thereby determined volumetric and areal capacitances for $10 \mathrm{mV} \mathrm{s}^{-1}$ scan rate were $34 \mathrm{~F} \mathrm{~cm}^{-3}$ and $171 \mathrm{mF} \mathrm{cm}^{-2}$, respectively. Moreover, the capacitance measured at $10 \mathrm{mV} \mathrm{s}^{-1}$ was retained $60 \%$ when scan rate increased to $100 \mathrm{mV} \mathrm{s}^{-1}$. Electrochemical impedance spectroscopy for PVA-LiCl and PVDF-HFP-TEA $\cdot \mathrm{BF}_{4}$ coated asymmetric supercapacitors indicates low equivalent series resistance, as shown in Fig. 3F.

The scan rate dependence of areal and linear capacitances for asymmetric supercapacitors using PVDF-HFP-TEA $\cdot \mathrm{BF}_{4}$ and PVA-LiCl electrolytes are compared in Fig. 4A. Although the organic electrolyte supercapacitor has a wider working voltage range, the specific capacitances were much lower than for the aqueous PVA-LiCl gel based supercapacitor. The reduced charge storage capability for the organic-electrolyte-based supercapacitor possibly results from larger ion size for this electrolyte.

A Ragone plot of areal energy density versus areal power density for asymmetric $\mathrm{rGO} / \mathrm{MnO}_{2}$ embedded yarn supercapacitors (based on the total surface area of the complete supercapacitor, including embedded yarns and PVDF-HFP$\mathrm{TEA} \cdot \mathrm{BF}_{4}$ or PVA- $\mathrm{LiCl}$ based electrolyte) is shown in Fig. 4B. Previously published data for yarn or fiber supercapacitors are included for comparison. The maximum measured areal energy densities of the presently investigated asymmetric supercapacitors based on PVDF-HFP-TEA $\cdot \mathrm{BF}_{4}$ organic gel and PVA$\mathrm{LiCl}$ based aqueous gel electrolytes are 30.1 and $43 \mu \mathrm{W} \mathrm{h} \mathrm{cm}{ }^{-2}$, respectively (normalized by total device including both two electrodes and electrolyte coating). These energy densities are higher than for previous 1D supercapacitors, which comprise (a) $\mathrm{CNT} / \mathrm{MnO}_{2}$ based stretchable asymmetric fibers $(18.9 \mu \mathrm{W}$ $\left.\mathrm{h} \mathrm{cm}{ }^{-2}\right),{ }^{22}$ (b) rGO/MnO $/$ PPy yarns $\left(9.2 \mu \mathrm{W} \mathrm{h} \mathrm{cm}{ }^{-2}\right),{ }^{11}$ (c) NiOH/ $\mathrm{MnO}_{2}$ asymmetric yarns $\left(10 \mu \mathrm{W} \mathrm{h} \mathrm{cm}{ }^{-2}\right){ }^{16}(\mathrm{~d})$ hollow rGO fibers $\left(6.8 \mu \mathrm{W} \mathrm{h} \mathrm{cm}{ }^{-2}\right),{ }^{10}$ (e) $\mathrm{MnO}_{2}$ nanosheet decorated asymmetric carbon fibers $\left(1.428 \mu \mathrm{W} \mathrm{h} \mathrm{cm}{ }^{-2}\right),{ }^{21}$ and (f) $\mathrm{MnO}_{2}$ coated stretchable, asymmetric CNT wires $\left(1.25 \mu \mathrm{W} \mathrm{h} \mathrm{cm}{ }^{-2}\right) .{ }^{19}$ Table 1 further compares the linear, areal, and volumetric energy

Table 1 Comparison of specific energies for present and prior-art fiber or yarn based asymmetric supercapacitors

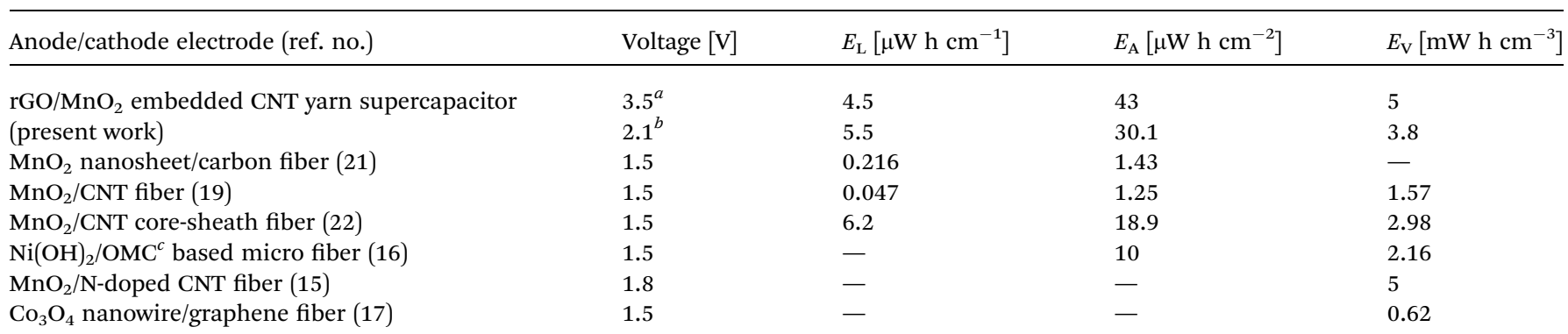

${ }^{a}$ TEA $\cdot \mathrm{BF}_{4}$-PC-(PVDF-co-HFP). ${ }^{b}$ LiCl-PVA gel electrolyte. ${ }^{c}$ OMC: ordered mesoporous carbon. 
A

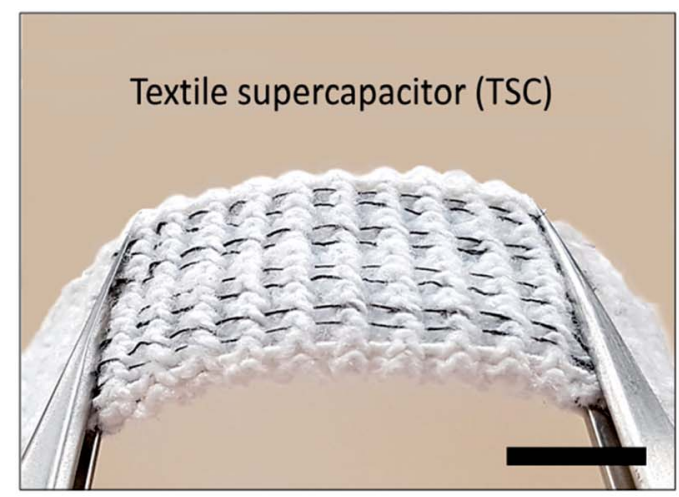

C

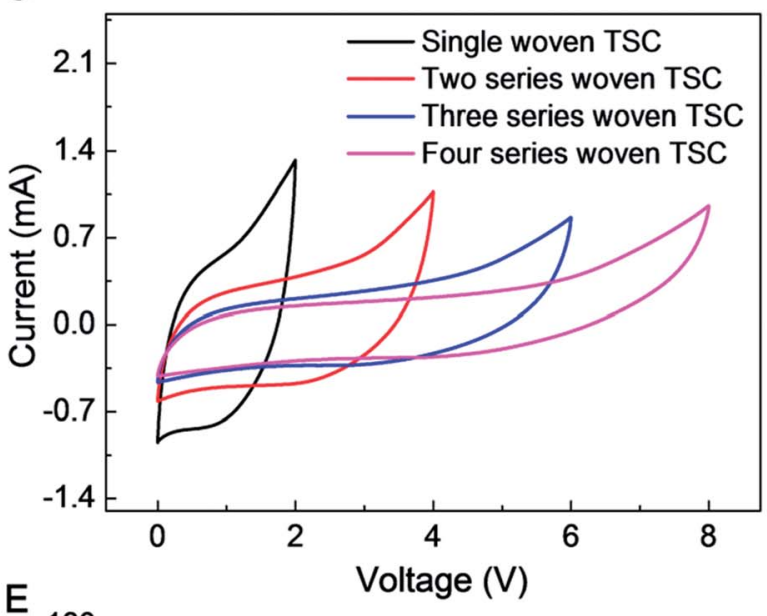

E

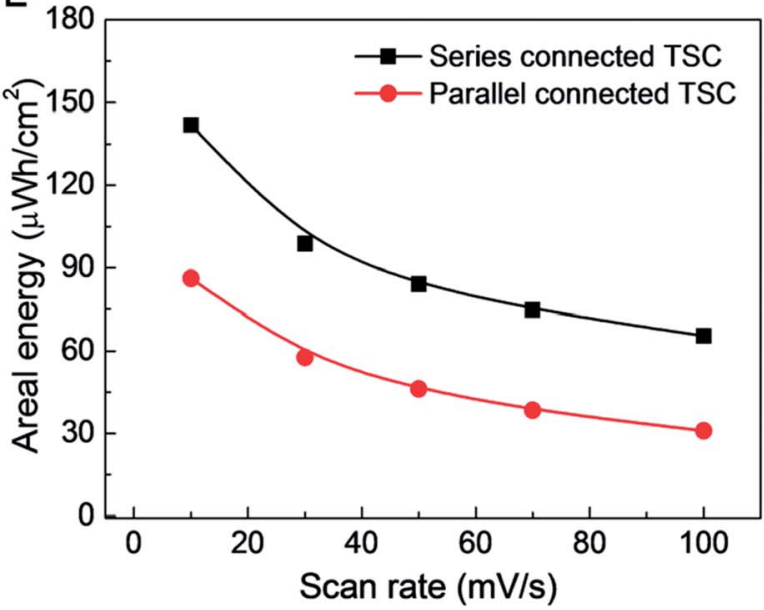

B

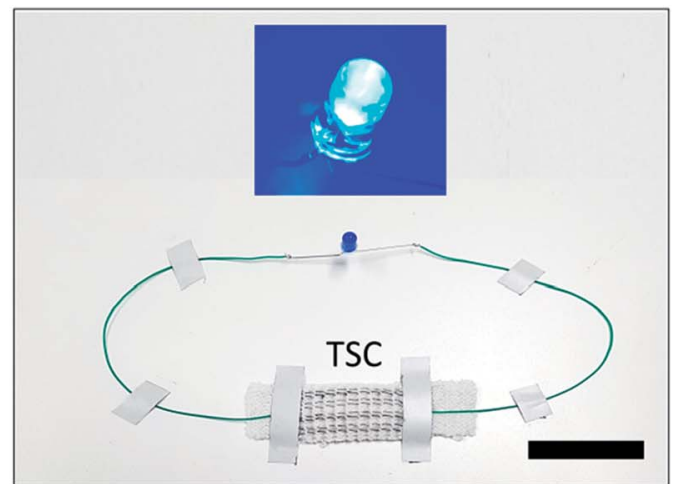

D

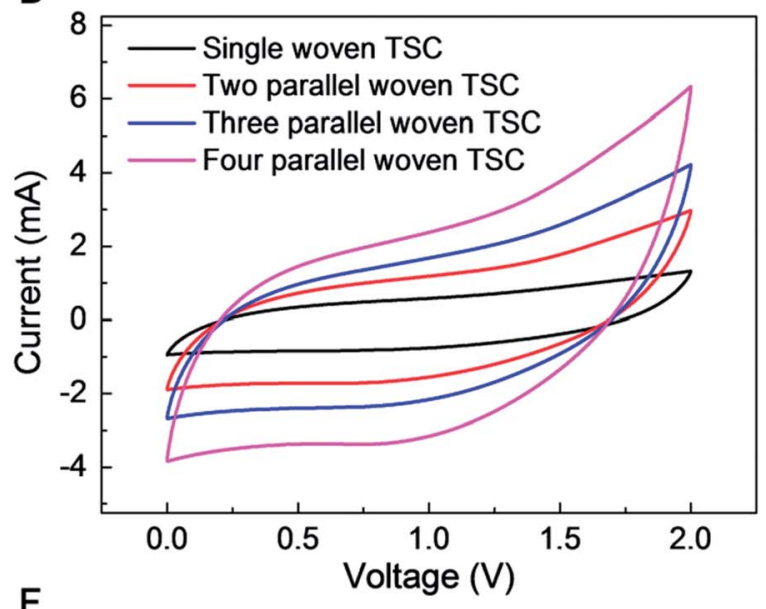

$\mathrm{F}$

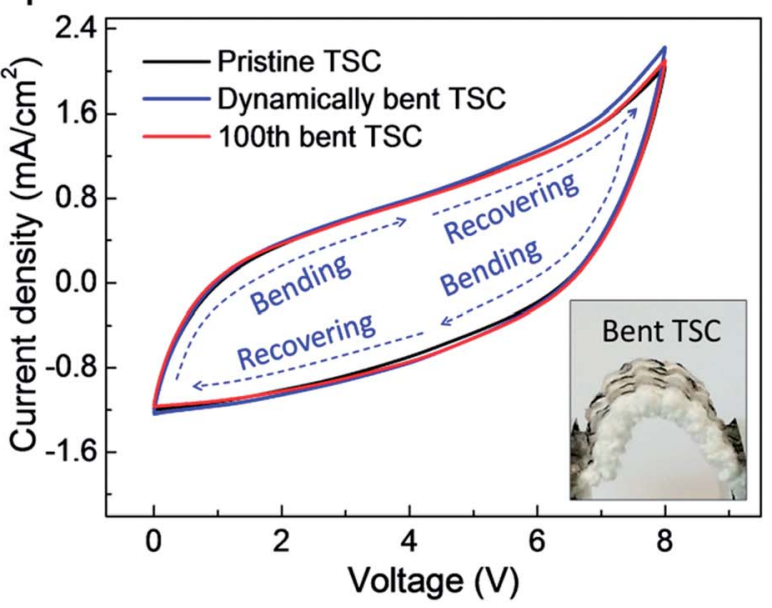

Fig. 5 The structure and performance of a textile supercapacitor (TSC) comprising $90 \mathrm{wt} \% \mathrm{rGO}$ embedded yarn anodes, $\mathrm{MnO}_{2}$ nanoparticle embedded yarn cathodes, and an aqueous PVA-LiCl gel electrolyte. (A) Optical image showing four asymmetric yarn supercapacitors sewn into a commercially available knitted textile, each comprising a $4 \mathrm{~cm}$-long rGO embedded anode and a $4 \mathrm{~cm}$-long $\mathrm{MnO}_{2}$ embedded cathode (scale bar $=1 \mathrm{~cm}$ ). (B) Blue LED lightening demonstration by asymmetric TSC (scale bar $=4 \mathrm{~cm}$ ). CV curves $\left(50 \mathrm{mV} \mathrm{s} \mathrm{s}^{-1} \mathrm{scan}\right.$ rate) for $(C)$ seriesconnected and (D) parallel-connected combinations of from 1 to 4 textile woven supercapacitors. (E) Energy density and specific capacitance of four series and parallel connected TSC versus scan rate. Textile surface area occupied by yarn electrodes is used for normalization. (F) The effects on the CV curves of dynamically and cyclically applied bending on a textile containing four series-connected supercapacitors. The CV curves (at $90 \mathrm{mV} \mathrm{s}^{-1}$ scan rate) before bending (black line), after 100 bending cycles (red line), and during bending (blue line) are shown.

densities of the presently reported asymmetric biscrolled yarn supercapacitors with results in the literature for other 1D asymmetric supercapacitors.
The goal of the following investigation of a textile based supercapacitor (TSC) is to show that supercapacitor performance does not degrade when supercapacitor yarns are 
deployed in a very stretchable fabric, even though the mechanical properties of the supercapacitor yarns and fabric are quite different. The commercial textile used had a mock rib structure, which can be used for a strong, elastic hem (such as edges of gloves). Eight $200 \mu \mathrm{m}$-diameter yarn electrodes (four biscrolled rGO anode yarns and four biscrolled $\mathrm{MnO}_{2}$ cathode yarns) were inserted into the textile (to replace eight elastic yarns in the mock rib stitch), as shown in Fig. 5A. Despite high loadings of guest powders (90 wt\% rGO for the anode yarn and $70 \mathrm{wt} \% \mathrm{MnO}_{2}$, for the cathode yarn) the yarn electrodes were sufficiently strong to be easily inserted into the textile, or even woven, without any additional treatment. Opposite ends of $4 \mathrm{~cm}$ long yarn electrodes were electrically connected to $180 \mu \mathrm{m}$ diameter $\mathrm{Cu}$ wires using silver paste, so that electrical connections could be made, and the woven anode and cathode yarn pairs of a supercapacitor were then jointly coated with aqueous PVA-LiCl gel electrolyte to complete the TSC.

Four asymmetric woven supercapacitors, each comprising alternating rGO embedded anode and $\mathrm{MnO}_{2}$ embedded cathode, were series or parallel direction connected to demonstrate scalability (a $2.5 \mathrm{~mm}$ gap between woven supercapacitors was used to prevent ionic currents between supercapacitors which would prohibit their use as series-connected devices). The series connected TSC demonstrated a sufficiently high voltage to power a blue $0.65 \mathrm{~W}$ LED for 300 seconds (Fig. S7 †), as shown in Fig. 5B.

CV curves for increasing numbers of in-series and in-parallel woven supercapacitors are shown in Fig. 5C and D, respectively. Series connection enabled voltages of up to $\sim 8 \mathrm{~V}$, while parallel connection enabled discharge current of up to $\sim 3.2 \mathrm{~mA}$ (at $1 \mathrm{~V}$ ). Based on these $\mathrm{CV}$ curves at $10 \mathrm{mV} \mathrm{s}^{-1}$ scan rate, the areal energy density and areal capacitance (normalized to total area occupied by the supercapacitors on the textile) for four seriesconnected TSC are $142 \mu \mathrm{W} \mathrm{h} \mathrm{cm} \mathrm{cm}^{-2}$ and $16 \mathrm{mF} \mathrm{cm}^{-2}$ and those for four parallel-connected TSC are $86.2 \mu \mathrm{W} \mathrm{h} \mathrm{cm}{ }^{-2}$ and $155 \mathrm{mF} \mathrm{cm}^{-2}$, respectively. Although the areal capacitance for series-connected TSC is much lower than for parallel connection (as is expected), much higher specific energy was obtained by enlarged operation voltage up to $8 \mathrm{~V}$ of series connection. The effects of dynamically and cyclically applied bending on textile containing four series-connected TSC around a mandrel (which results is a bending radius of $4 \mathrm{~mm}$ for the supercapacitors in the textile) are described in Fig. 5F. As shown here, 100 bending cycles or bending and releasing the textile during electrochemical cycling produced little change in CV scans.

\section{Conclusions}

In summary, asymmetric yarn supercapacitors which have high energy density and can be woven into textiles were investigated. The rGO embedded CNT yarn electrode which contains 90.1 wt\% rGO shows excellent charge storage capability (172 mF $\mathrm{cm}^{-2}$ ) which contributes to enhance overall electrochemical performance of the asymmetric supercapacitors. By combining with $\mathrm{MnO}_{2}$ embedded CNT yarn cathode, the wide voltage windows up to $2.1 \mathrm{~V}$ at aqueous electrolyte, and $3.5 \mathrm{~V}$ at organic electrolyte were obtained. The high areal energy densities measured from all-solid state asymmetric yarn supercapacitors were $30.1 \mu \mathrm{W} \mathrm{h} \mathrm{cm}{ }^{-2}$ and $43 \mu \mathrm{W} \mathrm{h} \mathrm{cm}^{-2}$ for PVA-LiCl and PVDFHFP-TEA $\cdot \mathrm{BF}_{4}$ gel electrolytes, respectively. Finally, the asymmetric supercapacitors could be woven into a knitted textile to make a TSC, and it shows a promising possibility to be used in electronic textiles by lighting a blue LED.

\section{Methods}

\section{Preparation of guest-embedded, biscrolled yarn electrochemical electrodes}

Narrow sheets of highly oriented carbon multiwalled nanotubes (MWNTs) were drawn from a MWNT forest $(\sim 400 \mu \mathrm{m}$ high and consisting of $\sim 12 \mathrm{~nm}$-diameter nanotubes containing $\sim 9$ walls), which was fabricated by a chemical vapor deposition. Nanoflakes of rGO dispersed in dimethylformamide (8 $\mathrm{mg} \mathrm{ml}^{-1}$ ) were ultrasonicated (for 1 hour at $150 \mathrm{~W}$ using a VCX750 ultrasonic processor from Sonics). After ultrasonication, the rGO dispersion was drop cast on a 5-layer-stack of these forest-drawn MWNT sheets, which were $\sim 3 \mathrm{~cm}$ wide and $20 \mathrm{~cm}$ long, and semi-dried for $\sim 20$ minutes. The drop casting and the semi-drying processes were repeated five times. Afterward, the $\mathrm{rGO} / \mathrm{CNT}$ sheet stack was converted to a biscrolled yarn by inserting $\sim 3000$ turns per meter (per sheet length) using an electrical motor. Commercially available $\mathrm{MnO}_{2}$ nanoparticles (rod shape with $\sim 30 \mathrm{~nm}$ diameter and $\sim 100 \mathrm{~nm}$ length from Sigma-Aldrich) were dispersed in ethanol (5 mg $\mathrm{ml}^{-1}$ ) and ultrasonicated, as done for the rGO. After drop casting the as-prepared $\mathrm{MnO}_{2}$ dispersion $\left(100 \mu \mathrm{l} \mathrm{cm}{ }^{-2}\right)$ on a four layer CNT sheet stack, the $\mathrm{MnO}_{2} / \mathrm{CNT}$ sheets were twisted to $\sim 5000$ turns per meter using an electrical motor to make the $\mathrm{MnO}_{2}$ embedded biscrolled yarn used as a supercapacitor cathode. One end of each electrode was connected to a $180 \mu \mathrm{m}$ diameter $\mathrm{Cu}$ wire using silver paste for electrochemical performance measurements.

\section{Supercapacitor assembly}

The aqueous PVA-LiCl gel electrolyte was prepared by heating a mixture of $3 \mathrm{~g}$ PVA $\left(M_{\mathrm{w}} 146000-186000\right)$ and $6 \mathrm{~g} \mathrm{LiCl}$ in $30 \mathrm{ml}$ deionized water at $90{ }^{\circ} \mathrm{C}$ for several hours. The PVDF-HFP$\mathrm{TEA} \cdot \mathrm{BF}_{4}$ gel electrolyte was prepared by drying a solution mixture of PVDF-HFP in acetone and $\mathrm{TEA} \cdot \mathrm{BF}_{4}$ in propylene carbonate (PC) on a slide glass for $\sim 3$ hours. The ratio of PVDF$\mathrm{HFP}$ in acetone and $\mathrm{TEA} \cdot \mathrm{BF}_{4}$ in $\mathrm{PC}$ was $4: 1$. The rGO embedded anode and the $\mathrm{MnO}_{2}$ embedded cathode were placed parallel and $\sim 100 \mu \mathrm{m}$ apart and then coated with PVA-LiCl (or PVDF-HFP-TEA $\cdot \mathrm{BF}_{4}$ ) gel electrolyte to complete asymmetric yarn supercapacitor fabrication. The TSC was made by removing textile threads from the mock rib structure and then using a needle to sew in their place $\mathrm{rGO} / \mathrm{CNT}$ embedded yarns and $\mathrm{MnO}_{2} / \mathrm{CNT}$ embedded yarns, which were then pairwise coated with the PVA/LiCl gel electrolyte. The chemicals for electrolyte synthesis were purchased from Sigma-Aldrich and Alfa-Aesar. 


\section{Characterization}

Cyclic voltammetry and chronopotentiometry measurements for all investigated supercapacitors were made using a electrochemical analyzer (CHI 627b, CH Instrument). Nyquist curves were measured using another electrochemical analyzer (Reference 600, Gamry Instrument). Scanning electron microscope images of biscrolled yarns were obtained using a Zeiss Supra 40 SEM. The length and weight of each electrode were measured using a digital Vernier caliper (500 series, Mitutoyo) and microbalance (XP6, Meter Toledo), respectively. Yarn diameters were obtained from optical images collected using a microscope with attached camera. X-Ray photoelectron spectroscopy data are collected from an X-ray photoelectron spectrometer (Theta Probe, Thermo Scientific Co.).

\section{Calculation of electrochemical performance}

The capacitances of supercapacitors were calculated from cyclic voltammograms using $C=I /(\mathrm{d} V / \mathrm{d} t)$, where $I$ is the discharge current and $\mathrm{d} V / \mathrm{d} t$ is the voltage scan rate. The specific areal capacitance for each electrode in a supercapacitor having equal anode and cathode capacitances was calculated using $C_{A}=4 C /$ $A$, where $A$ is the total surface area of the anode and cathode. The total length and total volume of electrodes were used to obtain linear capacitance and volumetric capacitance, respectively. The specific energy density was calculated from the equation $E=1 / 2 C V^{2}$. Resulting energy densities indicated in Fig. $4 \mathrm{~B}$ and Table 1 are for a complete supercapacitor (normalized by the total length, surface area, and volume of the embedded electrodes coated with electrolyte).

\section{Conflicts of interest}

There are no conflicts to declare.

\section{Acknowledgements}

This work was supported by the Creative Research Initiative Center for Self-powered Actuation in Korea, Basic Science Research Program through the National Research Foundation of Korea (NRF) funded by the Ministry of Education (NRF2017R1A6A3A04004987), and DGIST R\&D Program of Ministry of Science, ICT and Future Planning of Korea (17-NT-02). Support at the University of Texas at Dallas was provided by Air Force Office of Scientific Research grants AOARD-FA2386-131-4119 and FA9550-15-1-0089 and Robert A. Welch Foundation grant AT-0029. Additional support was from the Australian Research Council (DP110101073).

\section{References}

1 J. A. Lee, M. K. Shin, S. H. Kim, H. U. Cho, G. M. Spinks, G. G. Wallace, M. D. Lima, X. Lepro, M. E. Kozlov, R. H. Baughman and S. J. Kim, Nat. Commun., 2013, 4, 1970.

2 C. Choi, K. M. Kim, K. J. Kim, X. Lepro, G. M. Spinks, R. H. Baughman and S. J. Kim, Nat. Commun., 2016, 7, 13811.
3 C. Choi, J. M. Lee, S. H. Kim, S. J. Kim, J. Di and R. H. Baughman, Nano Lett., 2016, 16, 7677.

4 Y. Huang, M. Zhu, Y. Huang, Z. Pei, H. Li, Z. Wang, Q. Xue and C. Zhi, Adv. Mater., 2016, 28, 8344.

5 Y. Huang, W. S. Ip, Y. Y. Lau, J. Sun, J. Zen, N. S. S. Yeung, W. S. Ng, H. Li, Z. Pei, Q. Xue, Y. Wang, J. Yu, H. Hu and C. Zhi, ACS Nano, 2017, 11, 8953.

6 Y. Huang, M. Zhong, F. Shi, X. Liu, Z. Tang, Y. Wang, Y. Huang, H. Hou, X. Xie and C. Zhi, Angew. Chem., Int. Ed., 2017, 56, 1.

7 Q. Xue, J. Sun, Y. Huang, M. Zhu, Z. Pei, H. Li, Y. Wang, N. Li, H. Zhang and C. Zhi, Small, 2017, 13, 1701827.

8 C. Choi, J. A. Lee, A. Y. Choi, Y. T. Kim, X. Lepro, M. D. Lima, R. H. Baughman and S. J. Kim, Adv. Mater., 2013, 26, 2059.

9 J. Ren, C. Chen, X. Chen, Z. Cai, L. Qiu, Y. Wang, X. Zhu and H. Peng, Adv. Mater., 2013, 25, 1155.

10 G. Qu, J. Cheng, X. Li, D. Yuan, P. Chen, X. Chen, B. Wang and H. Peng, Adv. Mater., 2016, 28, 3646.

11 Y. Huang, H. Hu, Y. Huang, M. Zhu, W. Meng, C. Liu, Z. Pei, C. Hao, Z. Wang and C. Zhi, ACS Nano, 2015, 9, 4766.

12 J. Sun, Y. Huang, C. Fu, Z. Wang, Y. Huang, M. Zhu, C. Zhi and H. Hu, Nano Energy, 2016, 27, 230.

13 B. Wang, X. Fang, H. Sun, S. He, J. Ren, Y. Zhang and H. Peng, Adv. Mater., 2015, 27, 7854.

14 K. Wang, Q. Meng, Y. Zhang, Z. Wei and M. Miao, Adv. Mater., 2013, 25, 1494.

15 D. Yu, K. Goh, Q. Zhang, L. Wei, H. Wang, W. Jiang and Y. Chen, Adv. Mater., 2014, 26, 6790.

16 X. Dong, Z. Guo, Y. Song, M. Hou, J. Wang, Y. Wang and Y. Xia, Adv. Funct. Mater., 2014, 24, 3405.

17 X. Wang, B. Liu, R. Liu, Q. Wang, X. Hou, D. Chen, R. Wang and G. Shen, Angew. Chem., Int. Ed., 2014, 126, 1880.

18 B. Zheng, T. Huang, L. Kou, X. Zhao, K. Gopalsamy and C. Gao, J. Mater. Chem. A, 2014, 2, 9736.

19 P. Xu, B. Wei, Z. Cao, J. Zheng, K. Gong, F. Li, J. Yu, Q. Li, W. Lu, J. H. Byun, B. S. Kim, Y. Yan and T. W. Chou, ACS Nano, 2015, 9, 6088.

20 G. Sun, X. Zhang, R. Lin, J. Yang, H. Zhang and P. Chen, Angew. Chem., Int. Ed., 2015, 54, 4651.

21 N. Yu, H. Yin, W. Zhang, Y. Liu, Z. Tang and M. Q. Zhu, Adv. Energy Mater., 2016, 6, 1501458.

22 J. Yu, W. Lu, J. P. Smith, K. S. Booksh, L. Meng, Y. Huang, Q. Li, J. H. Byun, Y. Oh, Y. Yan and T. W. Chou, Adv. Energy Mater., 2017, 7, 1600976.

23 M. Lima, S. Fang, X. Lepró, C. Lewis, R. Ovalle-Robles, J. Carretero-González, E. Castillo-Martínez, M. Kozlov, J. Oh, N. Rawat, C. Haines, M. Haque, V. Aare, S. Stoughton, A. Zakhidov and R. Baughman, Science, 2011, 331, 51.

24 S. Gambhir, E. Murray, S. Sayyar, G. G. Wallace and D. L. Officer, Carbon, 2014, 76, 368.

25 X. Chen, L. Qiu, J. Ren, G. Guan, H. Lin, Z. Zhang, P. Chen, Y. Wang and H. Peng, Adv. Mater., 2013, 25, 6436.

26 H. Sun, X. You, J. Deng, X. Chen, Z. Yang, J. Ren and H. Peng, Adv. Mater., 2014, 26, 2868.

27 Y. Ma, P. Li, J. W. Sedloff, X. Zhang, H. Zhang and J. Liu, ACS Nano, 2015, 9, 1352. 
28 J. Ren, W. Bai, G. Guan, Y. Zhang and H. Peng, Adv. Mater., 2013, 25, 5965.

29 L. Kou, T. Huang, B. Zheng, X. Zhao, K. Gopalsamy, H. Sun and C. Gao, Nat. Commun., 2014, 5, 3754.
30 H. Wang, Z. Li, Y. Huang, Q. Li and X. Wang, J. Mater. Chem., 2010, 20, 3883.

31 J. A. Lee, Y. T. Kim, G. M. Spinks, D. Suh, X. Lepro, M. D. Lima, R. H. Baughman and S. J. Kim, Nano Lett., 2014, 14, 2664. 